\title{
Clinical utility of rivaroxaban in stroke prevention associated with nonvalvular atrial fibrillation - patient considerations
}

This article was published in the following Dove Press journal:

Journal of Blood Medicine

24 February 2014

Number of times this article has been viewed

\author{
Ingo Ahrens \\ Christoph Bode \\ Heart Center, University \\ of Freiburg, Department of \\ Cardiology and Angiology I, \\ Freiburg, Germany
}

Correspondence: Ingo Ahrens Heart Center, University of Freiburg, Department of Cardiology and Angiology I, Hugstetter Strasse 55, 79106 Freiburg, Germany Email ingo.ahrens@universitaetsherzzentrum.de

\begin{abstract}
The direct factor Xa inhibitor rivaroxaban was the first within the group of orally available direct factor Xa inhibitors to gain clinical approval for oral anticoagulation in patients with nonvalvular atrial fibrillation in 2011. The -xabans, as drugs from the group of oral direct factor Xa inhibitors are often referred to, comprise currently three drugs (apixaban, edoxaban, and rivaroxaban) with clinical approval for a variety of clinical indications that require oral anticoagulation therapy. The number of patients with nonvalvular atrial fibrillation requiring long-term oral anticoagulation therapy for the prevention of stroke and systemic embolism is predicted to increase up to five times by 2050. Many of these patients will be within the elderly and aging population that is at a higher risk of stroke and also at a higher risk for bleeding events. This requires novel options for efficient and safe oral anticoagulation, and rivaroxaban is one of the novel oral anticoagulants that have been shown to be at least as effective as vitamin $\mathrm{K}$ antagonists in patients with nonvalvular atrial fibrillation. Furthermore, like all of the novel oral anticoagulants, rivaroxaban provides a significant reduction in intracerebral hemorrhage compared with vitamin $\mathrm{K}$ antagonists such as warfarin. The clinical utility of oral anticoagulation with rivaroxaban in patients with nonvalvular atrial fibrillation is discussed here, along with special patient considerations, including impaired renal function, switching from a vitamin $\mathrm{K}$ antagonist, and patients with concomitant acute coronary syndrome.
\end{abstract}

Keywords: rivaroxaban, factor Xa, stroke, intracranial bleeding, vitamin K antagonist, atrial fibrillation, acute coronary syndrome, triple therapy, BAY 59-7939, DX9065a, DU-176b

\section{Atrial fibrillation and risk of stroke}

Atrial fibrillation is the most common heart rhythm disorder seen in daily clinical practice. ${ }^{1}$ Currently, the prevalence of atrial fibrillation in the adult population is estimated at $1 \%-2 \%$, and increases to up to $10 \%$ in patients older than 80 years of age. ${ }^{2}$ With the aging of the population, atrial fibrillation is expected to increase by at least up to 2.5 times by $2050 .^{3}$ The risk of stroke in patients with atrial fibrillation who are not on chronic oral anticoagulation therapy used to be estimated by the $\mathrm{CHADS}_{2}$ score system ( $0-6$ points), which predicts an annual stroke rate of $1.9 \%$ ( 0 points) up to $18.2 \%$ (6 points). ${ }^{4} \mathrm{~A}$ refined scoring system, the $\mathrm{CHA}_{2}-\mathrm{DS}_{2}$-VASc score, takes additional factors that increase the risk for stroke into account, such as sex and age older than 74 years. This scoring system spans from 0 to 9 points and predicts annual stroke rates of $0 \%, 1.3 \%, 2.2 \%, 3.2 \%, 4 \%, 6.7 \%, 9.8 \%, 9.6 \%, 6.7 \%$, and $15.2 \%$, corresponding to $0,1,2,3,4,5,6,7,8$, and 9 points on the $\mathrm{CHA}_{2}-\mathrm{DS}_{2}-$ VASc. ${ }^{5}$ According to the $\mathrm{CHA}_{2}-\mathrm{DS}_{2}$-VASc score, only patients with lone atrial fibrillation and those under the age of 65 years may be considered as being at low risk for stroke and therefore may not need oral anticoagulation. ${ }^{6}$ 


\section{Factor Xa: a target for novel oral anticoagulants}

Factor Xa and factor Va are components of the prothrombinase complex, which catalyzes the generation of thrombin. Formation of the prothrombinase complex regulates thrombin production and is therefore a key component of the coagulation system. ${ }^{7}$ Further, recent evidence indicates that factor $\mathrm{Xa}$ itself has an activating effect on factor $\mathrm{V}$ and thereby facilitates assembly of the prothrombinase complex. ${ }^{8}$ Given the critical role of factor $\mathrm{Xa}$ in the coagulation cascade, it does appear as an ideal target for anticoagulant drugs. The successful clinical development of parenteral indirect factor $\mathrm{Xa}$ inhibitors, such as the low molecular weight heparins and fondaparinux, endorsed the concept of developing direct factor Xa inhibitors as anticoagulant drugs.

The recent clinical breakthrough in the development of synthetic direct and specific factor Xa inhibitors as novel oral anticoagulants as a substitute for vitamin $\mathrm{K}$ antagonists is the result of an intensive and long-lasting (at least 20 years) development period that started with the discovery of DX-9065a (Daiichi Sankyo Inc., Tokyo, Japan), a synthetic direct orally available factor Xa inhibitor. ${ }^{8}$ Due to its low oral bioavailability, further clinical development of DX-9065a initially focused on parenteral application. ${ }^{9-11}$ Nearly a decade later, the clinical research turned back into developing DX-9065a as an orally available novel anticoagulant drug by replacing an amidine moiety in DX-9065a to improve oral bioavailability, thereby generating the new compound DU-176b. ${ }^{12}$ This compound is now known as edoxaban (Lixiana ${ }^{\mathbb{}}$; Daiichi Sankyo Co., Ltd, Tokyo, Japan), and has successfully completed major Phase III clinical trials in thromboembolism ${ }^{13}$ and atrial fibrillation. ${ }^{14}$ However, in the meantime, a synthetic direct and orally available novel factor Xa inhibitor (BAY 59-7939) was discovered. ${ }^{15}$ This compound was later named rivaroxaban $\left(\right.$ Xarelto $^{\circledR}$; Janssen Pharmaceuticals, Inc., Titusville, NJ, USA) and rapidly proceeded to successful clinical development into the first oral direct factor Xa inhibitor with clinical approval in $2008 .{ }^{7}$

\section{Pharmacology of rivaroxaban}

Rivaroxaban is a small-molecule direct factor Xa inhibitor with high oral bioavailability. Compared with other serine proteases, the affinity of rivaroxaban for factor Xa is $>10,000$ times higher, thereby guaranteeing potent inhibition of generation of thrombin from prothrombin, which is mediated downstream of factor $\mathrm{X}$ activation by the prothrombinase complex. ${ }^{16}$ After oral intake, rivaroxaban reaches peak plasma concentrations within 3-4 hours. Elimination of rivaroxaban follows a dual mode, ie, two thirds of the drug is metabolized by the liver and one third is eliminated unchanged by the kidney. This leads to a mean terminal half-life for rivaroxaban of 7-11 hours. ${ }^{16}$

\section{Anticoagulation with rivaroxaban in patients with atrial fibrillation}

Rivaroxaban was the first direct oral factor Xa inhibitor to gain clinical approval for prevention of stroke and systemic embolism in patients with nonvalvular atrial fibrillation in 2011, and the second novel oral anticoagulant that gained approval in this indication (the first novel oral anticoagulant with clinical approval in patients with nonvalvular atrial fibrillation being the oral direct thrombin inhibitor dabigatran etexilate, approved in 2010).

The major clinical trial leading to the approval of rivaroxaban in atrial fibrillation was the multicenter, randomized, double-blind, double-dummy, event-driven ROCKET AF (The Rivaroxaban Once Daily Oral Direct Factor Xa Inhibition Compared with Vitamin K Antagonism for Prevention of Stroke and Embolism Trial in Atrial Fibrillation). ${ }^{17}$

In ROCKET AF, a total of 14,264 patients with nonvalvular atrial fibrillation and a moderate to high risk of stroke were randomized to oral rivaroxaban $20 \mathrm{mg}$ once daily (15 mg once daily in patients with creatinine clearance of 30-49 $\mathrm{mL}$ per minute) or to dose-adjusted warfarin. The International Normalized Ratio (INR) target in the warfarin group was 2.0-3.0, and mock INR values were generated in the group of patients receiving rivaroxaban. The mean $\mathrm{CHADS}_{2}$ score of all patients randomized in ROCKET AF was 3.5 and the median time for INR values within the therapeutic range (INR 2.0-3.0) was 58\%. ${ }^{17}$ The primary efficacy endpoint was the composite of ischemic or hemorrhagic stroke and systemic embolism. The primary safety endpoint was the composite of major and nonmajor clinically relevant bleeding. Major bleeding was defined as clinically overt bleeding associated with a drop in hemoglobin of at least $2 \mathrm{~g} / \mathrm{dL}$, or transfusion of two or more units of packed red blood cells or whole blood, or bleeding at a critical site such as intracranial, intraspinal, intraocular, pericardial, intra-articular, intramuscular with compartment syndrome, retroperitoneal, or a fatal outcome.

In the per-protocol population, the primary endpoint occurred in $1.7 \%$ of patients per year in the rivaroxabantreated group and in $2.2 \%$ per year in the warfarin-treated group ( $P<0.001$ for noninferiority of rivaroxaban). In the as-treated safety population, the primary endpoint occurred at a similar rate ( $P=0.01$ for superiority). ${ }^{17}$ The primary safety endpoint of major and clinically relevant nonmajor bleeding 
occurred in $14.9 \%$ per year of the rivaroxaban-treated patients and $14.5 \%$ of the warfarin-treated patients $(P=0.44)$. Major bleeding occurred at a rate of $3.6 \%$ in rivaroxaban-treated patients and $3.4 \%$ in warfarin-treated patients $(P=0.58)$. While major bleeding from a gastrointestinal site was more common in rivaroxaban-treated patients $(3.2 \%$ versus $2.2 \%$ in warfarin-treated patients, $P<0.001)$, there was a significant reduction in intracranial hemorrhage on rivaroxaban $(0.5 \%$ versus $0.7 \%$ on warfarin, $P=0.02) .{ }^{17}$

\section{Reduced risk for intracranial bleeding}

Intracranial bleeding is the most feared complication of chronic oral anticoagulation. In the decades when oral anticoagulation was restricted to vitamin $\mathrm{K}$ antagonists only, the risk was estimated to be as high as $1 \%$ per year, with approximately $60 \%$ of such events being fatal. ${ }^{18} \mathrm{~A}$ common feature of all novel oral anticoagulants is that they significantly reduce the risk for intracranial hemorrhage, thereby providing a safer bleeding risk profile compared with vitamin K antagonists. ${ }^{19-21}$ Data from large real-world registries on rivaroxaban in patients with atrial fibrillation will help to determine whether the favorable bleeding risk profile regarding intracranial hemorrhage that has been observed in the large randomized clinical trials translates in everyday clinical practice. At least one large registry of rivaroxaban in routine clinical practice is currently recruiting patients (NCT01947959). ${ }^{22}$

In the ROCKET AF trial, intracranial hemorrhage occurred in only $0.5 \%$ per year in the rivaroxaban-treated patients and in $0.7 \%$ per year in the warfarin-treated patients $(P=0.02){ }^{17}$ In the smaller J-ROCKET AF trial in 1,280 Japanese patients with nonvalvular atrial fibrillation, less intracranial bleeding was also observed with rivaroxaban (five patients, $0.8 \%$ ) compared with warfarin (ten patients, 1.6\%), thereby supporting the transferability of the global ROCKET AF study results to the Japanese population. ${ }^{23}$

\section{Patients with impaired renal function}

Among patients with nonvalvular atrial fibrillation, those with impaired renal function do have a higher risk for stroke or systemic embolism, ${ }^{24}$ and at the same time they are also at a higher risk for bleeding events according to the HAS-BLED score. ${ }^{25}$ This underscores the need for safe and efficient oral anticoagulation in patients with nonvalvular atrial fibrillation and chronic kidney disease. In ROCKET AF, patients with reduced renal function (creatinine clearance $30-49 \mathrm{~mL}$ per minute) randomized to rivaroxaban received a reduced dose of $15 \mathrm{mg}$ daily instead of $20 \mathrm{mg}$ daily. ${ }^{17} \mathrm{In}$ a subgroup analysis of ROCKET AF in patients with reduced renal function (creatinine clearance $30-49 \mathrm{~mL}$ per minute), the primary endpoint of stroke or systemic embolism occurred in $2.32 \%$ of patients per year in the group treated with rivaroxaban $15 \mathrm{mg}$ daily compared with $2.77 \%$ per year in the warfarin-treated group. ${ }^{26}$ The primary safety endpoint of major or nonmajor clinically relevant bleeding was also similar between patients treated with rivaroxaban $15 \mathrm{mg}$ daily and those treated with warfarin ( $17.82 \%$ versus $18.28 \%$ per year for the rivaroxaban-treated and warfarin-treated patients, respectively). In addition, fatal bleeding occurred less frequently with rivaroxaban $15 \mathrm{mg}$ daily in patients with impaired renal function (creatinine clearance 30-49 mL per minute) compared with warfarin $(0.28 \%$ versus $0.74 \%$ per year for rivaroxaban and warfarin, respectively, $P=0.047){ }^{26}$

Current prescription information on rivaroxaban for nonvalvular atrial fibrillation recommends a $15 \mathrm{mg}$ daily dose in patients with moderate impairment of renal function (creatinine clearance $30-49 \mathrm{~mL}$ per minute). Further, rivaroxaban $15 \mathrm{mg}$ daily should be used with caution in patients with a creatinine clearance of $15-29 \mathrm{~mL}$ per minute and should not be used in those with a creatinine clearance below $15 \mathrm{~mL}$ per minute. However, it remains to be determined whether a further reduction of rivaroxaban from $15 \mathrm{mg}$ daily to $10 \mathrm{mg}$ daily would preserve the benefit to risk profile in patients with nonvalvular atrial fibrillation and more severely impaired renal function of 15-29 mL per minute. Data from a small pharmacokinetic and pharmacodynamic study in volunteers suggest that patients with a creatinine clearance of $15-29 \mathrm{~mL}$ per minute receiving a $10 \mathrm{mg}$ daily dose are exposed to similar plasma rivaroxaban concentrations as those exposed to a dose of $20 \mathrm{mg}$ daily. ${ }^{27}$ This needs to be further explored by future clinical studies with repeat doses of $10 \mathrm{mg}$ daily rivaroxaban in patients with nonvalvular atrial fibrillation and more severely impaired renal function (creatinine clearance 15-29 mL per minute).

\section{Switching patients on vitamin K antagonists to rivaroxaban}

More than half of the randomized patients with nonvalvular atrial fibrillation $(7,897,55.4 \%)$ in the ROCKET AF trial were already on chronic oral anticoagulant therapy. These patients were identified if they had used vitamin K antagonists for at least 6 weeks at the time of screening for enrollment into ROCKET AF and were defined as "vitamin K antagonist-experienced." ${ }^{\prime 17}$ A recent subgroup analysis from 
the ROCKET AF trial examined whether there are differences in clinical outcome between vitamin $\mathrm{K}$ antagonistexperienced and vitamin $\mathrm{K}$ antagonist-naïve patients who were randomized in ROCKET AF. In vitamin K antagonistexperienced patients who were randomized to rivaroxaban therapy, treatment with rivaroxaban was initiated when the INR value was below 3.0. Vitamin $\mathrm{K}$ antagonist-experienced patients had a trend for a generally lower rate of stroke and cardiovascular events than vitamin $\mathrm{K}$ antagonist-naïve patients. At the same time, rivaroxaban reduced the rate of hemorrhagic stroke regardless of whether the patients were vitamin $\mathrm{K}$ antagonist-experienced or vitamin $\mathrm{K}$ antagonistnaïve. ${ }^{28}$ There were more bleeding events with rivaroxaban in vitamin $\mathrm{K}$ antagonist-experienced patients within the first 7 days of initiation of the study drug. However, after 30 days, there was no difference in bleeding rates between rivaroxaban and warfarin in vitamin $\mathrm{K}$ antagonist-experienced patients and there were fewer bleeding events with rivaroxaban in vitamin $\mathrm{K}$ antagonist-naïve patients. ${ }^{28}$ Therefore, it appears to be a safe clinical strategy to switch a vitamin K antagonistexperienced patient with nonvalvular atrial fibrillation from a vitamin K antagonist to rivaroxaban. However, the patient's individual clinical situation and economic considerations also have to be taken into account when a decision to switch a vitamin $\mathrm{K}$ antagonist-experienced patient with nonvalvular atrial fibrillation to rivaroxaban therapy is made.

Vitamin $\mathrm{K}$ antagonist-naïve patients with nonvalvular atrial fibrillation appear to have fewer bleeding complications when they are initiated on rivaroxaban therapy. ${ }^{28}$ Further, the reduced rate of intracranial bleeds that was observed with rivaroxaban therapy in the ROCKET AF trial supports the initiation of rivaroxaban instead of a vitamin $\mathrm{K}$ antagonist in vitamin $\mathrm{K}$ antagonist-naïve patients with nonvalvular atrial fibrillation.

\section{Atrial fibrillation and coronary artery disease}

Among the 14,264 patients with nonvalvular atrial fibrillation enrolled in the ROCKET AF trial, there were 2,468 (17.3\%) who had a prior myocardial infarction. This indicates a high prevalence of coronary artery disease in patients with atrial fibrillation and is supported by other clinical databases reporting a prevalence of coronary artery disease of $6.4 \%$ for all-comer patients with nonvalvular atrial fibrillation in the Japanese population. ${ }^{29}$ In ROCKET AF, rivaroxabantreated patients with a prior myocardial infarction showed a nonsignificant reduction of ischemic cardiac events compared with warfarin-treated patients. The common clinical situation that (according to current concepts) requires both dual antiplatelet therapy and anticoagulation arises if patients with atrial fibrillation requiring chronic oral anticoagulation are undergoing percutaneous coronary intervention with stent implantation. These patients have an increased risk for allcause mortality, ischemic stroke, and intracranial bleeding. ${ }^{30}$ There is currently only one published study available, ie, WOEST (What is the Optimal antiplatElet and anticoagulant therapy in patients with oral anticoagulation and coronary StenTing) that addressed the challenging clinical situation of patients on chronic oral anticoagulation undergoing percutaneous coronary intervention. In this study, 573 patients were randomized to either aspirin + clopidogrel + a vitamin $\mathrm{K}$ antagonist (triple therapy) or to clopidogrel + a vitamin $\mathrm{K}$ antagonist (dual therapy). The rates of major and minor bleeding events were doubled in the triple therapy group compared with the dual therapy group (31.3\% versus $14 \%$ for triple therapy versus dual therapy respectively, $P<0.001$ ) while at the same time there were no significant differences in thrombotic events between triple therapy and dual therapy in this trial. ${ }^{31}$ However, only one quarter of patients randomized in the WOEST trial was presenting with an acute coronary syndrome, which has important clinical implications because patients with acute coronary syndromes are more likely to have thrombotic events following percutaneous coronary intervention than patients with stable coronary artery disease.

Thinking about possible future antithrombotic strategies in patients with atrial fibrillation and acute coronary syndromes, it is important to discuss the recently published ATLAS ACS 2 TIMI 51 (Anti-Xa Therapy to Lower Cardiovascular Events in Addition to Standard Therapy in Subjects With Acute Coronary Syndrome ACS 2-Thrombolysis In Myocardial Infarction 51) trial. This trial examined whether addition of low dose rivaroxaban $2.5 \mathrm{mg}$ or $5 \mathrm{mg}$ twice daily to standard dual antiplatelet therapy (aspirin + clopidogrel or ticlopidine) was superior to standard dual antiplatelet therapy alone in patients with a recent acute coronary syndrome (noting that these patients did not suffer from atrial fibrillation). In ATLAS ACS 2 TIMI 51, addition of rivaroxaban $2.5 \mathrm{mg}$ twice daily significantly reduced cardiovascular and allcause death $(2.7 \%$ versus $4.1 \%$ and $2.9 \%$ versus $4.5 \%$ in the rivaroxaban-treated and placebo-treated patients respectively, both $P=0.002) .{ }^{32}$ On the other hand, it did increase the risk of TIMI major bleeding and intracranial hemorrhage $(1.8 \%$ versus $0.6 \%, P<0.001$, and $0.4 \%$ versus $0.2 \%, P=0.04$, for rivaroxaban-treated and placebo-treated patients, respectively). Importantly, addition of rivaroxaban did not increase 
the rate of fatal bleeding $(0.1 \%$ versus $0.2 \%, P=0.45$ for rivaroxaban versus placebo respectively). ${ }^{32}$

Based on the results of the ATLAS ACS 2 TIMI 51 clinical trial and subgroup analysis of patients from the ROCKET AF trial with prior myocardial infarction and the clinical data from the WOEST trial, it appears to be a reasonable approach to investigate whether a combination of dual antiplatelet therapy (aspirin + a P2Y12 receptor antagonist) + low dose rivaroxaban (2.5 mg twice daily) or single antiplatelet therapy (P2Y12 receptor antagonist) + reduced dose rivaroxaban (15 mg once daily) is superior to the current standard triple therapy of aspirin + a P2Y12 inhibitor + a vitamin K antagonist. This is currently being examined in the openlabel, randomized PIONEER AF-PCI (A Study Exploring Two Strategies of Rivaroxaban and One of Oral Vitamin K Antagonist in Patients With Atrial Fibrillation Who Undergo Percutaneous Coronary Intervention) clinical trial in 2110 patients (NCT01830543). ${ }^{33}$

\section{Summary and recommendations}

The novel oral anticoagulants are generally associated with a significantly reduced risk for intracranial hemorrhage compared with warfarin. This is especially important in patients with newly diagnosed nonvalvular atrial fibrillation who require chronic oral anticoagulation for prevention of stroke and systemic embolism. Among the direct oral factor Xa inhibitors, rivaroxaban was the first compound that gained clinical approval in 2011 for stroke prevention in nonvalvular atrial fibrillation based on the results of ROCKET AF, a landmark clinical trial in 14,264 patients with nonvalvular atrial fibrillation. Currently unsolved challenges in daily clinical practice include the optimal treatment and dosing of novel oral anticoagulants in patients with severely impaired renal function and concomitant anticoagulation therapy in patients with atrial fibrillation and acute coronary syndromes that require dual antiplatelet therapy. In summary:

- rivaroxaban is used at a single daily dose of $20 \mathrm{mg}$ for prevention of stroke and systemic embolism in patients with nonvalvular atrial fibrillation

- in patients with impaired renal function (creatinine clearance of 30-49 mL per minute), a reduced dose of $15 \mathrm{mg}$ rivaroxaban once daily is recommended

- in patients with a creatinine clearance of 15-29 mL per minute, the reduced dose of $15 \mathrm{mg}$ rivaroxaban once daily should be used with caution; there are currently no clinical data available that confirm the risk/benefit profile of an even lower rivaroxaban dose of $10 \mathrm{mg}$ once daily in patients with nonvalvular atrial fibrillation and more severely reduced renal function (creatinine clearance 15-29 $\mathrm{mL}$ per minute)

- switching patients from vitamin $\mathrm{K}$ antagonists to rivaroxaban is safe and does preserve the benefit/risk profile of rivaroxaban observed in clinical trials in patients with nonvalvular atrial fibrillation

- patients with atrial fibrillation and coronary artery disease undergoing percutaneous coronary intervention and therefore requiring dual antiplatelet therapy are at increased risk for bleeding events when a full dose of oral anticoagulant is added; on the other hand, these patients are at increased risk for stroke if antithrombotic therapy is limited to dual platelet inhibition only; whether a reduced dose novel oral anticoagulant added to dual antiplatelet therapy or an intermediate dose novel oral anticoagulant added to single antiplatelet therapy could be a viable strategy for these patients is currently being assessed in clinical trials.

\section{Disclosure}

IA has received speaker's honoraria from Bayer Healthcare, Lilly, Sanofi aventis, and Daiichi Sankyo. CB has received speaker's honoraria from Bayer Healthcare, Merck, Astra-Zeneca, Daiichi Sankyo, and Sanofi aventis.

\section{References}

1. Shah AJ, Liu X, Jadidi AS, Haissaguerre M. Early management of atrial fibrillation: from imaging to drugs to ablation. Nat Rev Cardiol. 2010;7(6):345-354

2. Ahrens I, Bode C. Rivaroxaban for stroke prevention in atrial fibrillation and secondary prevention in patients with a recent acute coronary syndrome. Future Cardiol. 2012;8(4):533-541.

3. Go AS, Hylek EM, Phillips KA, et al. Prevalence of diagnosed atrial fibrillation in adults: national implications for rhythm management and stroke prevention: the AnTicoagulation and Risk Factors in Atrial Fibrillation (ATRIA) Study. JAMA. 2001;285(18):2370-2375.

4. Gage BF, Waterman AD, Shannon W, Boechler M, Rich MW, Radford MJ. Validation of clinical classification schemes for predicting stroke: results from the National Registry of Atrial Fibrillation. JAMA. 2001;285(22):2864-2870.

5. Lip GY, Frison L, Halperin JL, Lane DA. Identifying patients at high risk for stroke despite anticoagulation: a comparison of contemporary stroke risk stratification schemes in an anticoagulated atrial fibrillation cohort. Stroke. 2010;41(12):2731-2738.

6. Coppens M, Eikelboom JW, Hart RG, et al. The CHA2DS2-VASc score identifies those patients with atrial fibrillation and a CHADS2 score of 1 who are unlikely to benefit from oral anticoagulant therapy. Eur Heart $J$. 2013;34(3):170-176

7. Ahrens I, Lip GY, Peter K. New oral anticoagulant drugs in cardiovascular disease. Thromb Haemost. 2010;104(1):49-60.

8. Hara T, Yokoyama A, Ishihara H, Yokoyama Y, Nagahara T, Iwamoto M. DX-9065a, a new synthetic, potent anticoagulant and selective inhibitor for factor Xa. Thromb Haemost. 1994;71(3):314-319.

9. Murayama N, Tanaka M, Kunitada S, et al. Tolerability, pharmacokinetics, and pharmacodynamics of DX-9065a, a new synthetic potent anticoagulant and specific factor Xa inhibitor, in healthy male volunteers. Clin Pharmacol Ther. 1999;66(3):258-264. 
10. Ahrens I, Peter K, Bode C. [Factor Xa-inhibition in interventional cardiology]. Hamostaseologie. 2007;27(5):328-332. German.

11. Morishima Y, Tanabe K, Terada Y, Hara T, Kunitada S. Antithrombotic and hemorrhagic effects of DX-9065a, a direct and selective factor Xa inhibitor: comparison with a direct thrombin inhibitor and antithrombin III-dependent anticoagulants. Thromb Haemost. 1997;78(5): 1366-1371.

12. Furugohri T, Isobe K, Honda Y, et al. DU-176b, a potent and orally active factor Xa inhibitor: in vitro and in vivo pharmacological profiles. J Thromb Haemost. 2008;6(9):1542-1549.

13. Buller HR, Decousus H, Grosso MA, et al. Edoxaban versus warfarin for the treatment of symptomatic venous thromboembolism. $N$ Engl J Med. 2013;369(15):1406-1415.

14. Giugliano RP, Ruff CT, Braunwald E, et al. Edoxaban versus warfarin in patients with atrial fibrillation. $N$ Engl J Med. 2013;369(22): 2093-2104.

15. Roehrig S, Straub A, Pohlmann J, et al. Discovery of the novel antithrombotic agent 5-chloro-N-(\{(5S)-2-oxo-3- [4-(3-oxomorpholin4-yl)phenyl]-1,3-oxazolidin-5-yl $\}$ methyl)thiophene-2-carboxamide (BAY 59-7939): an oral, direct factor Xa inhibitor. J Med Chem. 2005;48(19):5900-5908.

16. Perzborn E, Roehrig S, Straub A, Kubitza D, Misselwitz F. The discovery and development of rivaroxaban, an oral, direct factor $\mathrm{Xa}$ inhibitor. Nat Rev Drug Discov. 2011;10(1):61-75.

17. Patel MR, Mahaffey KW, Garg J, et al. Rivaroxaban versus warfarin in nonvalvular atrial fibrillation. $N$ Engl J Med. 2011;365(10): 883-891.

18. Hart RG, Boop BS, Anderson DC. Oral anticoagulants and intracranial hemorrhage. Facts and hypotheses. Stroke. 1995;26(8):1471-1477.

19. Dentali F, Riva N, Crowther M, Turpie AG, Lip GY, Ageno W. Efficacy and safety of the novel oral anticoagulants in atrial fibrillation: a systematic review and meta-analysis of the literature. Circulation. 2012;126(20):2381-2391.

20. Wasserlauf G, Grandi SM, Filion KB, Eisenberg MJ. Meta-analysis of rivaroxaban and bleeding risk. Am J Cardiol. 2013;112(3): 454-460.

21. Chatterjee S, Sardar P, Biondi-Zoccai G, Kumbhani DJ. New oral anticoagulants and the risk of intracranial hemorrhage: traditional and Bayesian meta-analysis and mixed treatment comparison of randomized trials of new oral anticoagulants in atrial fibrillation. JAMA Neurol. October 28, 2013. [Epub ahead of print.]

22. Bayer. Study of Rivaroxaban Use and Potential Adverse Outcomes in Routine Clinical Pratice (Germany). Available from: http://clinicaltrials.gov/ct2/show/NCT01947959. NLM identifier: NCT01947959. Accessed January 8, 2014.
23. Hori M, Matsumoto M, Tanahashi N, et al. Rivaroxaban vs warfarin in Japanese patients with atrial fibrillation - the J-ROCKET AF study. Circ J. 2012;76(9):2104-2111.

24. Hart RG, Eikelboom JW, Ingram AJ, Herzog CA. Anticoagulants in atrial fibrillation patients with chronic kidney disease. Nat Rev Nephrol. 2012;8(10):569-578.

25. Pisters R, Lane DA, Nieuwlaat R, de Vos CB, Crijns HJ, Lip GY. A novel user-friendly score (HAS-BLED) to assess 1-year risk of major bleeding in patients with atrial fibrillation: the Euro Heart Survey. Chest. 2010;138(5):1093-1000.

26. Fox KA, Piccini JP, Wojdyla D, et al. Prevention of stroke and systemic embolism with rivaroxaban compared with warfarin in patients with non-valvular atrial fibrillation and moderate renal impairment. Eur Heart J. 2011;32(19):2387-2394.

27. Kubitza D, Becka M, Mueck W, et al. Effects of renal impairment on the pharmacokinetics, pharmacodynamics and safety of rivaroxaban, an oral, direct Factor Xa inhibitor. Br J Clin Pharmacol. 2010;70(5): $703-712$.

28. Mahaffey KW, Wojdyla D, Hankey GJ, et al. Clinical outcomes with rivaroxaban in patients transitioned from vitamin $\mathrm{K}$ antagonist therapy: a subgroup analysis of a randomized trial. Ann Intern Med. 2013;158(12):861-868.

29. Senoo K, Suzuki S, Sagara K, et al. Coronary artery diseases in Japanese patients with nonvalvular atrial fibrillation. J Cardiol. September 23, 2013. [Epub ahead of print.]

30. Pilgrim T, Kalesan B, Zanchin T, et al. Impact of atrial fibrillation on clinical outcomes among patients with coronary artery disease undergoing revascularisation with drug-eluting stents. Euro Intervention . 2013;8(9):1061-1071.

31. Dewilde WJ, Oirbans T, Verheugt FW, et al. Use of clopidogrel with or without aspirin in patients taking oral anticoagulant therapy and undergoing percutaneous coronary intervention: an open-label, randomised, controlled trial. Lancet. 2013;381(9872):1107-1115.

32. Mega JL, Braunwald E, Wiviott SD, et al. Rivaroxaban in patients with a recent acute coronary syndrome. $N$ Engl J Med. 2012;366(1):9-19.

33. Janssen Scientific Affairs, LLC. A Study Exploring Two Strategies of Rivaroxaban (JNJ39039039; BAY-59-7939) and One of Oral Vitamin K Antagonist in Patients With Atrial Fibrillation Who Undergo Percutaneous Coronary Intervention (PIONEER AF-PCI). Available from: http:// clinicaltrials.gov/show/NCT01830543. NLM identifier: NCT01830543. Accessed January 8, 2014.
Journal of Blood Medicine

\section{Publish your work in this journal}

The Journal of Blood Medicine is an international, peer-reviewed, open access, online journal publishing laboratory, experimental and clinical aspects of all topics pertaining to blood based medicine including but not limited to: Transfusion Medicine; Blood collection, Donor issues, Transmittable diseases, and Blood banking logistics; Immunohematology; Artificial and alternative

\section{Dovepress}

blood based therapeutics; Hematology; Biotechnology/nanotechnology of blood related medicine; Legal aspects of blood medicine; Historical perspectives. The manuscript management system is completely online and includes a very quick and fair peer-review system. Visit http://www.dovepress.com/ testimonials.php to read real quotes from published authors. 